
\title{
R Reserach S Suare \\ Coronavirus disease 2019 screening in adult patients hospitalized due to pneumonia in a single hospital in Japan.
}

Junpei Komagamine ( $\sim$ junpei0919@yahoo.co.jp )

National Hospital Organization Tochigi Medical Center https://orcid.org/0000-0002-5899-4760

Taku Yabuki

National Hospital Organization Tochigi Medical Center

\section{Short Report}

Keywords: COVID-19, epidemiology, pneumonia

Posted Date: June 13th, 2020

DOI: https://doi.org/10.21203/rs.3.rs-33316/v1

License: (c) (i) This work is licensed under a Creative Commons Attribution 4.0 International License.

Read Full License 


\section{Abstract}

Background: In Japan, the number of patients with coronavirus disease (COVID-19) has increased since the first case was confirmed in January 2020, reaching more than ten thousand cases as of April 30 , 2020. Nonetheless, a true prevalence of COVID-19 in Japan remains unknown due to testing restrictions and a lack of epidemiological research for severe acute respiratory syndrome coronavirus 2 (SARS-CoV2).

Methods: We investigated 37 consecutive pneumonia patients hospitalized in our hospital located in Utsunomiya, Japan, from February 25 to May 16, 2020. Both nasopharyngeal swabs and sputum were obtained and sent for SARS-CoV-2 PCR testing. The percentage of positive results for SARS-CoV-2 in the screened patients was determined.

Results: None of the patients reported in this study had contact with COVID-19 patients. Of the 37 screened patients, none had positive SARS-CoV-2 test results. None were clinically diagnosed with COVID19.

Conclusions: Our findings suggest that COVID-19 was not prevalent in this region during this period. Further studies are needed to determine an accurate number or prevalence of COVID-19 in other regions of Japan.

\section{Introduction}

Since the first case of severe acute respiratory syndrome coronavirus 2 (SARS-CoV-2) was confirmed in December 2019 in China [1], coronavirus disease 2019 (COVID-19) has rapidly spread worldwide [2]. In Japan, the number of patients with COVID-19 has increased since the first case was confirmed in January 2020, reaching more than ten thousand cases as of April 30, 2020 [3]. However, an accurate number of COVID-19 patients in Japan remains unknown due to testing restrictions for SARS-CoV-2. Moreover, the prevalence of COVID-19 among pneumonia patients, patients with common cold symptoms, and asymptomatic patients in Japan is also unknown. Therefore, we investigated the prevalence of COVID-19 in hospitalized pneumonia patients who were not confirmed for COVID-19 before admission to our hospital.

\section{Methods}

\section{Study design and setting}

A retrospective observational study was conducted by using electronic medical records in a single hospital. Our hospital is designated to provide care for adult patients with COVID-19 in Utsunomiya. Therefore, adult patients with confirmed or suspected COVID-19 in this area are first referred to our hospital. Utsunomiya is a city with a population of approximately 0.5 million and is located $100 \mathrm{~km}$ north of Tokyo. As of May 18, 2020, only twenty COVID-19 cases (including non-pneumonia or asymptomatic 
cases) have been confirmed, and the rate of positive results using SARS-CoV-2 polymerase chain reaction (PCR) testing is 2.5\% (20/816) in this city [4]. The prevalence of COVID-19 among hospitalized pneumonia patients who were not confirmed for COVID-19 before admission to our hospital was determined.

\section{Screening and inclusion criteria}

From February 25 to May 16, 2020, 42 adult patients were hospitalized in our hospital due to pneumonia. Of those, five patients were already diagnosed with pneumonia due to COVID-19 and transferred from other hospitals in this area. Three pneumonia patients with COVID-19 reported close contact with COVID-19 patients or a visit to a pandemic area outside Utsunomiya within two weeks before the onset of pneumonia. In the other two COVID-19 patients, the exposure history associated with COVID-19 remains unknown (detailed information is shown in supplementary file). We included the remaining 37 pneumonia patients who were screened on admission for exposure history and symptoms of COVID-19. Both nasopharyngeal swabs and sputum were obtained from all but one patient and sent to the Utsunomiya Public Health Center Laboratory for SARS-CoV-2 PCR testing. These investigations were not performed for one patient who was diagnosed with aspiration pneumonia because sudden respiratory failure occurred after the apparent aspiration episode. In patients with an undetermined cause of pneumonia, SARS-CoV-2 PCR testing was repeated at least twice 24 hours apart.

\section{Data collection}

Information on patient age, sex, Charlson Comorbidity Index score [5], past medical history, vital signs, clinical symptoms, laboratory data, and causes of the pneumonia was extracted from the electronic medical records. Causes of the pneumonia were determined based on physicians' diagnosis that was documented in the medical records.

\section{Statistical analysis}

Descriptive statistics were used to report the characteristics of the included patients. The percentage of positive results for SARS-CoV-2 in the screened patients was determined. These analyses were performed using STATA version 15 (LightStone, Tokyo, Japan).

\section{Results}

Of the 37 screened pneumonia patients, the mean patient age was 79.1 years old, $45.9 \%$ were women, and the mean Charlson Comorbidity Index score was 2.7 (Table). None of the patients reported having contact with COVID-19 patients, but one patient reported visiting a pandemic area within two weeks 
before the onset pneumonia. None of the patients had positive SARS-CoV-2 PCR testing results, although about three-fifths of patients were tested twice for SARS-CoV-2.

Table. Characteristics of the 37 consecutive pneumonia patients screened for SARS-CoV-2. 


\begin{tabular}{|c|c|}
\hline & Screened patients $(n=37)$ \\
\hline Mean age, years (SD) & $79.1(14.4)$ \\
\hline Female sex, n (\%) & $17(45.9)$ \\
\hline Nursing care resident, n (\%) & $7(18.9)$ \\
\hline Mean Charlson Comorbidity Index (SD) & $2.7(1.9)$ \\
\hline \multicolumn{2}{|c|}{ Exposure within 14 days before onset of pneumonia, $n$ (\%) } \\
\hline Contact with COVID-19 patients & $0(0.0)$ \\
\hline Any contact with sick patients & $3(8.1)$ \\
\hline Travel to foreign countries & $0(0.0)$ \\
\hline Visit to pandemic areas in Japan & $1(2.7)$ \\
\hline \multicolumn{2}{|l|}{ Past medical history, n (\%) } \\
\hline Dementia & $13(35.1)$ \\
\hline Ischemic heart disease & $3(8.1)$ \\
\hline Diabetes & $11(29.7)$ \\
\hline Hypertension & $18(48.7)$ \\
\hline Mean time to presentation from onset, days (SD) & $4.8(3.4)$ \\
\hline \multicolumn{2}{|l|}{ Presentation symptom, n (\%) } \\
\hline Fever & $28(75.7)$ \\
\hline Cough & $17(45.9)$ \\
\hline Dyspnea & $17(45.9)$ \\
\hline Sputum & $12(32.4)$ \\
\hline Throat pain & $3(8.1)$ \\
\hline Rhinorrhea & $3(8.1)$ \\
\hline \multicolumn{2}{|l|}{ Cause of pneumonia ${ }^{a}$} \\
\hline Aspiration pneumonia & $12(32.4)$ \\
\hline Typical pneumonia (bacterial) & $11(29.7)$ \\
\hline Atypical pneumonia & $8(21.6)$ \\
\hline Probable viral pneumonia & $1(2.7)$ \\
\hline Unknown cause & $4(10.8)$ \\
\hline
\end{tabular}


'These were based on physicians' diagnosis that was documented in the medical records.

COVID-19, coronavirus disease 2019; SD, standard deviation.

The most common cause of pneumonia was aspiration pneumonia $(n=12,32.4 \%)$, followed by typical pneumonia $(n=11,29.7 \%)$ and atypical pneumonia $(n=8,21.6 \%)$. None were clinically diagnosed with COVID-19. One pneumonia patient who presented with fever, dry cough, throat pain, and rhinorrhea for 10 days was clinically diagnosed with probable viral pneumonia based on the course of symptoms and no response of antimicrobial therapy. Results of SARS-CoV-2 PCR testing for this patient were negative twice, and results of tests for Mycoplasma pneumoniae, Chlamydophila pneumonia, and Legionella pneumophila were also negative.

\section{Discussion}

To our knowledge, this was the first study to determine the prevalence of COVID-19 among hospitalized pneumonia patients in Japan. During the study period, there were no positive results for SARS-CoV-2 in pneumonia patients screened in our hospital. The positive rate of SARS-CoV-2 in this study is much lower than that of women admitted for delivery in New York City [6], residents of a large homeless shelter in Boston [7], and symptomatic healthcare workers in England [8]; however, it is similar to that of the asymptomatic Icelandic population [9]. Our findings suggest that COVID-19 was not prevalent in Utsunomiya during the study period and that the risk for COVID-19 is still low without visiting pandemic areas or having contact with COVID-19 patients. Moreover, these results support the low number of confirmed COVID-19 patients and low rate of positive results for SARS-CoV-2 PCR testing in Utsunomiya. Thus, in this area, it is important to control the inflow of COVID-19 from other pandemic areas of Japan, including Tokyo. To prevent the spread of COVID-19 in Japan, an accurate number or prevalence of patients with COVID-19 in other parts of Japan also needs to be determined.

\section{Limitations}

First, the limitations of our study are the retrospective study design and small sample size. Second, limited sensitivity of SARS-CoV-2 PCR testing for COVID-19 might underestimate the prevalence of COVID-19 in pneumonia patients. Thirds, the accurate prevalence of COVID-19 in hospitalized pneumonia patients in Utsunomiya remains unknown because the number of pneumonia patients hospitalized in other hospitals in this area during the study period was not investigated.

\section{Conclusions}


Our findings suggest that COVID-19 was not prevalent in Utsunomiya during the study period and support the low number of confirmed COVID-19 patients in this area. Further studies are needed to determine the accurate prevalence of COVID-19 in Japan.

\section{Declarations}

Ethics approval and consent to participate

This research was approved by the Medical Ethical Committee of the National Hospital Organization Tochigi Medical Center (No. 2020-4). The need for individual informed consent was formally waived by the Medical Ethics Committee of the National Hospital Organization Tochigi Medical Center because we collected deidentified data without contacting the patients. However, per the Japanese Ethical Guidelines, we displayed an opt-out statement in the waiting room and webpage of the hospital to inform patients about the study and provide the opportunity for patients to refuse to allow the use of their data.

Competing interests

The authors declare no competing interests.

\section{References}

1. Zhu N, Zhang D, Wang W, et al. A novel coronavirus from patients with pneumonia in China, 2019. N Engl J Med 2020;382:727-33.

2. World Health Organization. Coronavirus disease (COVID-19) pandemic. https://www.who.int/emergencies/diseases/novel-coronavirus-2019 (lastly accessed on 20 May 2020).

3. Ministry of Health, Labour and Welfare. About coronavirus disease (COVID-19). https://www.mhlw.go.jp/stf/seisakunitsuite/bunya/newpage_00032.html (lastly accessed on 30 April 2020).

4. Utsunomiya city. The outbreak situation of new coronavirus infectious disease in Utsunomiya city [Japanese]. https://www.city.utsunomiya.tochigi.jp/kurashi/kenko/kansensho/etc/1023506.html (lastly accessed on 20 May 2020).

5. Charlson ME, Pompei P, Ales KL, MacKenzie CR. A new method of classifying prognostic comorbidity in longitudinal studies: development and validation. J Chron Dis 1987;40(5):373-83.

6. Sutton D, Fuchs K, D'Alton M, Goffman D. Universal screening for SARS-CoV-2 in women admitted for delivery. N Engl J Med 2020. doi:10.1056/NEJMc2009316.

7. Baggett TP, Keyes H, Sporn N, Gaeta JM. Prevalence of SSARS-CoV-2 infection in residents of a large homeless shelter in Boston. JAMA 2020. doi:10.1101/2020.04.12.20059618.

8. Hunter E, Price DA, Murphy E, et al. First experience of COVID-19 screening of health-care workers in England. Lancet 2020. doi:10.1016/S0140-6736(20)30970-3. 
9. Gudbjartsson DF, Helgason A, Jonsson H, et al. Spread of SARS-CoV-2 in the Icelandic population. N Engl J Med 2020. doi:10.1056/NEJMoa2006100.

\section{Supplementary Files}

This is a list of supplementary files associated with this preprint. Click to download.

- JGFMCOVID19supplementaryfile.pdf 